\title{
Abscopal effect following radiation monotherapy in breast cancer: A case report
}

\author{
AYAKA AZAMI $^{1}$, NOBUYASU SUZUKI ${ }^{1}$, YUSUKE AZAMI ${ }^{2}$, ICHIRO SETO $^{2}$, ATAI SATO $^{1}$, YOSHINAO TAKANO $^{1}$, \\ TSUYOSHI ABE ${ }^{1}$, YASUSHI TERANISHI ${ }^{1}$, KAZUNOSHIN TACHIBANA $^{3}$ and TOHRU OHTAKE ${ }^{3}$ \\ Departments of ${ }^{1}$ Surgery and ${ }^{2}$ Radiation Therapy, Southern Tohoku Research Institute for Neuroscience, \\ Southern Tohoku General Hospital, Koriyama, Fukushima 963-8563; ${ }^{3}$ Department of Breast Surgery, \\ Fukushima Medical University School of Medicine, Fukushima, Fukushima 960-1295, Japan
}

Received February 14, 2018; Accepted July 19, 2018

DOI: $10.3892 / \mathrm{mco} .2018 .1677$

\begin{abstract}
Radiotherapy has been found to be valuable for the control and eradication of local foci in various malignant tumors. The abscopal effect is determined as a systemic antitumor response at a distance from the irradiation site invoked by local irradiation. We herein present an extremely rare case of breast cancer in a 64-year-old woman, in whom the abscopal effect was observed after radiotherapy induced an antitumor response in all metastatic lesions, without any combination therapy. The patient was admitted to our hospital complaining of a breast mass and pain at the left hip, and was diagnosed with breast cancer with multiple bone, lung and lymph node metastases. She received treatment with local radiotherapy delivered to the breast tumor and some of the bone metastases but did not receive chemotherapy due to her poor performance status. However, 10 months after radiotherapy, spontaneous regression was observed, not only within the irradiated field, but also in the non-irradiated areas. All signs of cancer throughout the body disappeared, and the patient's performance status drastically improved. To the best of our knowledge, there have been no reports of advanced breast cancer cases in which the abscopal effect was observed after
\end{abstract}

Correspondence to: Dr Ayaka Azami, Department of Surgery, Southern Tohoku Research Institute for Neuroscience, Southern Tohoku General Hospital, 7-115 Yatsuyamada, Koriyama, Fukushima 963-8563, Japan

E-mail: azamiayaka0223@gmail.com

Abbreviations: CT, computed tomography; CEA, carcinoembryonic antigen; CA15-3, carbohydrate antigen 15-3; PET, positron emission tomography; FDG, ${ }^{18} \mathrm{~F}$-fluorodeoxyglucose; ECOG PS, Eastern Cooperative Oncology Group performance status; ICD, immunogenic cell death; CTL, cytotoxic T-lymphocyte; CTLA4, cytotoxic T-lymphocyte antigen; QOL, quality of life Key words: breast cancer, radiotherapy, abscopal effect, antitumor response, spontaneous regression

Key words: breast cancer, radiotherapy, abscopal effect, anti-tumor response, spontaneous regression radiation monotherapy; therefore, this case report is extremely rare and highly valuable.

\section{Introduction}

Radiotherapy is employed to control and eradicate foci of disease in several malignant tumors. The response to radiation may not only be evident locally, but also invoked by local irradiation at a distance from the irradiated site, which is referred to as the abscopal effect (1). The abscopal effect was first described by Mole (2) in 1953 and is a very rare antitumor immune response. It has been reported that this effect may be observed in patients receiving radiotherapy for several malignant tumors, including malignant melanoma $(3,4)$, lymphoma (5), renal cell carcinoma (6), hepatocellular carcinoma $(7,8)$, lung cancer $(9,10)$ and uterine cervical carcinoma (11). We herein report an extremely rare case of a breast cancer patient with multiple bone, lung and lymph node metastases who responded dramatically to local radiotherapy, without administration of systemic therapy.

\section{Case report}

A 64-year-old postmenopausal female Japanese patient was admitted with a tumor of the right breast for 1 year. The patient presented to Southern Tohoku General Hospital (Koriyama, Japan) with difficulty walking due to pain at the left hip joint and lower extremity. On physical examination, a sizeable mass was found to occupy almost the entire right breast, with ulceration of the overlying skin.

Core needle biopsy and subsequent histological examination revealed invasive ductal breast carcinoma (Fig. 1A), with positive estrogen receptor expression (Fig. 1B), mild positive progesterone receptor expression (Fig. 1C), negative for HER2 expression. Ki-67 proliferative index was 55\% (Fig. 1D). The results of the laboratory tests were within the normal limits. The serum levels of carcinoembryonic antigen (CEA) and carbohydrate antigen (CA)15-3were increased to $24.7 \mathrm{ng} / \mathrm{ml}$ and $81.3 \mathrm{U} / \mathrm{ml}$, respectively (normal range is less than $5 \mathrm{ng} / \mathrm{ml}$ or $28 \mathrm{U} / \mathrm{ml}$, respectively). A single fixed mass, $10 \mathrm{~cm}$ in greatest diameter, with ulceration of the overlying skin, was found to occupy almost the entire right breast, with palpable hard 

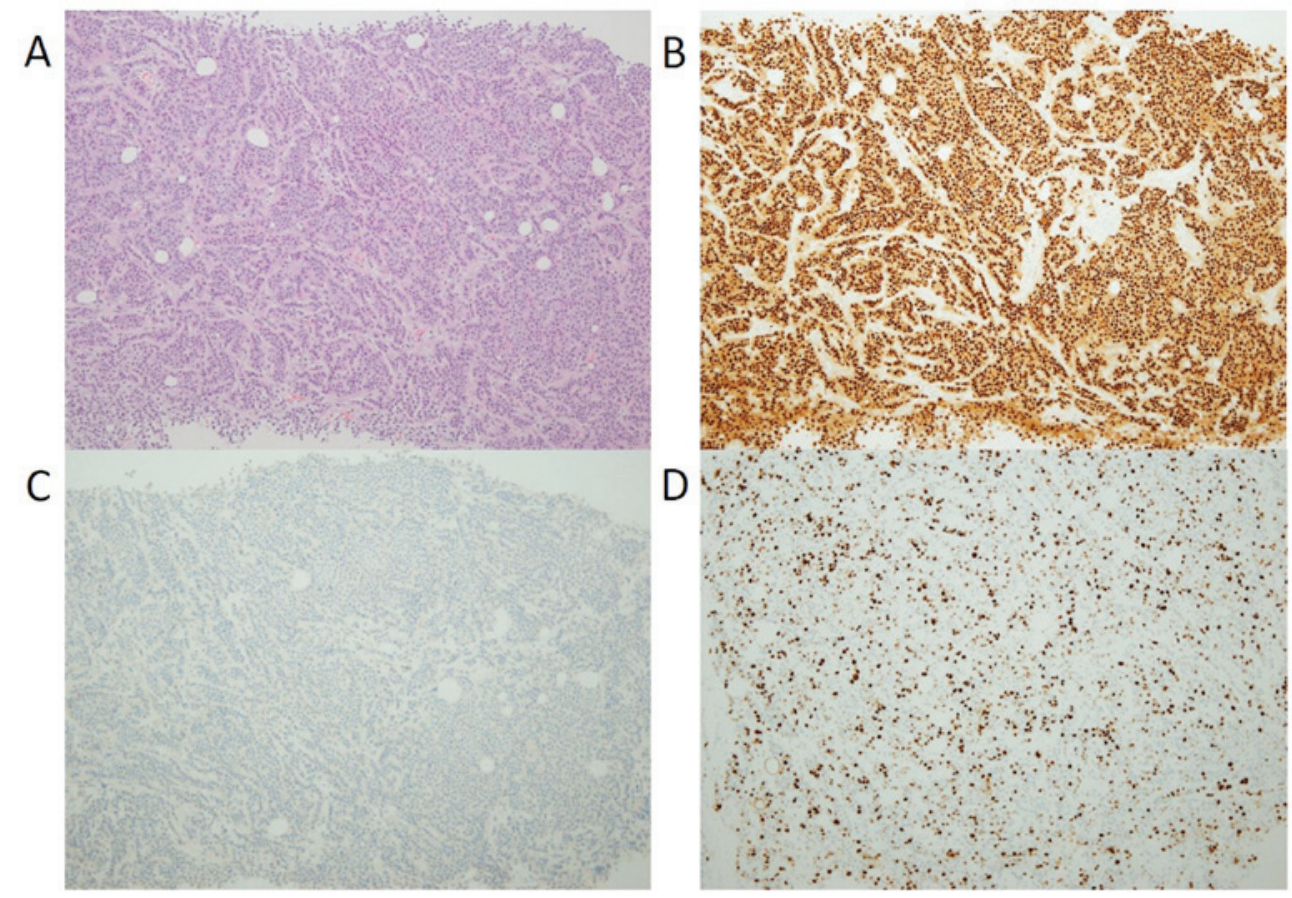

Figure 1. Histological examination of the breast tumor. (A) Hematoxylin and eosin staining; (B) ER staining; (C) PgR staining; (D) Ki-67 staining. Magnification, x50. ER, estrogen receptor; PgR, progesterone receptor.

ipsilateral axillary nodes. A computed tomography scan revealed a sizeable mass with contrast enhancement in the right breast. Positron emission tomography (PET) indicated abnormal ${ }^{18} \mathrm{~F}$-fluorodeoxyglucose (FDG) uptake in the right breast, with multiple lung and bone metastases, and multiple positive nodes in the lung, right axilla, right supraclavicular area and the mediastinum (Fig. 2A). Pelvic X-ray revealed osteolytic lesions in the left side of the femur, lumbar vertebrae and sacrum.

The patient received localized palliative radiotherapy to the breast tumor and some of the bone metastases that were associated with intense pain and high fracture risk. The radiation dose was $60 \mathrm{~Gy}$ to the right breast (Fig. 3A), 28 Gy to the left femur (Fig. 3B) and 39 Gy to the lumbar vertebrae and sacrum (Fig. 3C), at a daily 2-Gy fractionated dose. The patient received no systemic therapy, such as chemotherapy, molecular targeted therapy or hormone therapy. The treatment plan was designed with a palliative intent, rather than as radical medical therapy, as the patient was almost bedridden and could not move unassisted, which suggested that her performance status score was 3-4, as determined by the Eastern Cooperative Oncology Group (ECOG).

A total of 10 months following radiotherapy, the patient had a minor accident at home and was diagnosed with a left intertrochanteric femoral fracture. A PET scan for assessment prior to femoral open reduction internal fixation revealed a dramatic disease remission, determined as complete response, not only in all the irradiated sites, but rather in all the sites originally exhibiting abnormal FDG uptake (Fig. 2B). The serum levels of CEA and CA15-3 were also normalized. The wide skin ulcer of the breast cancer had also disappeared, along with complete lack of abnormal FDG uptake. The patient was able to walk unassisted, and her ECOG performance status recovered to 0 following femoral open reduction internal fixation and rehabilitation. A total of 3 months later, hormone therapy with aromatase inhibitor (anastrozole $1 \mathrm{mg} /$ body/day) was initiated, as the PET scan revealed mild progression of the metastases at the sternum. A total of 21 months later, bone metastases were stable disease and there were no metastases in internal organ (April 2018 last follow up).

\section{Discussion}

Radiotherapy is a valuable local treatment modality for malignant tumors. However, spontaneous remission in the metastatic lesions outside the irradiated field may be observed, which is referred to as the abscopal effect. The abscopal effect was first described by Mole (2) in 1953 and is a very rare antitumor immune response. It has been reported that the abscopal effect may be observed in cases of radiotherapy for several malignant tumors, including malignant melanoma $(3,4)$, lymphoma (5), renal cell carcinoma (6), hepatocellular carcinoma (7,8), lung cancer $(9,10)$ and uterine cervical carcinoma (11). Radiotherapy was also reported to be effective for a primary unknown bone metastasis, as the abscopal effect was observed when the metastatic lesions were irradiated (12). The clinical reports on the abscopal effect following radiation monotherapy are particularly rare. Furthermore, to the best of our knowledge, there has been no case report thus far of the abscopal effect in a breast cancer case with extensive metastases treated by radiotherapy alone.

The mechanisms underlying the abscopal effect were described by Golden et al (13). The mechanisms of radiation-induced tumor cell death include immunogenic cell death (ICD) in particular. Following radiation exposure, tumor cells are eliminated and tumor antigens are released. These 
A

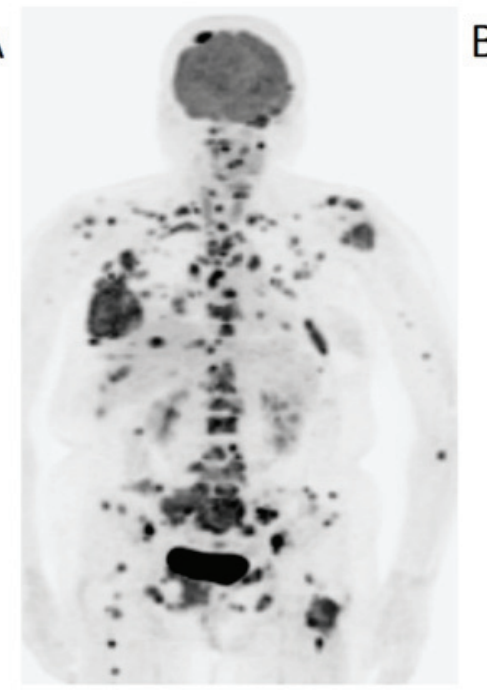

B

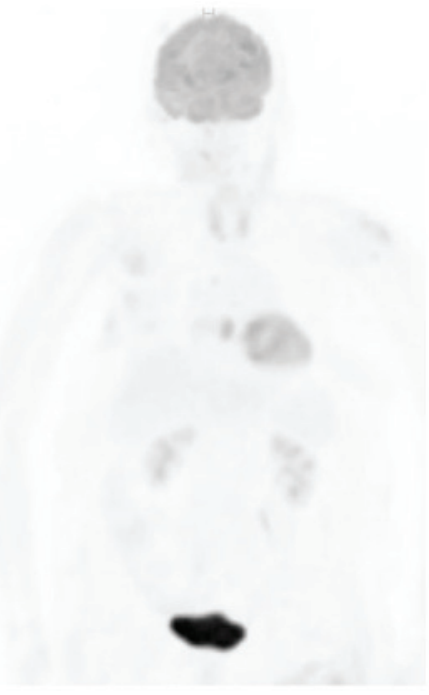

Figure 2. (A) PET scan demonstrating abnormal FDG uptake in the right breast, multiple lung lesions, bone lesions, and multiple nodes in the lung, right axilla, supraclavicular region and mediastinum. (B) PET scan showing dramatic disease remission, defined as complete response, 10 months following local radiotherapy. PET, positron emission tomography; FDG, fluorodeoxyglucose.
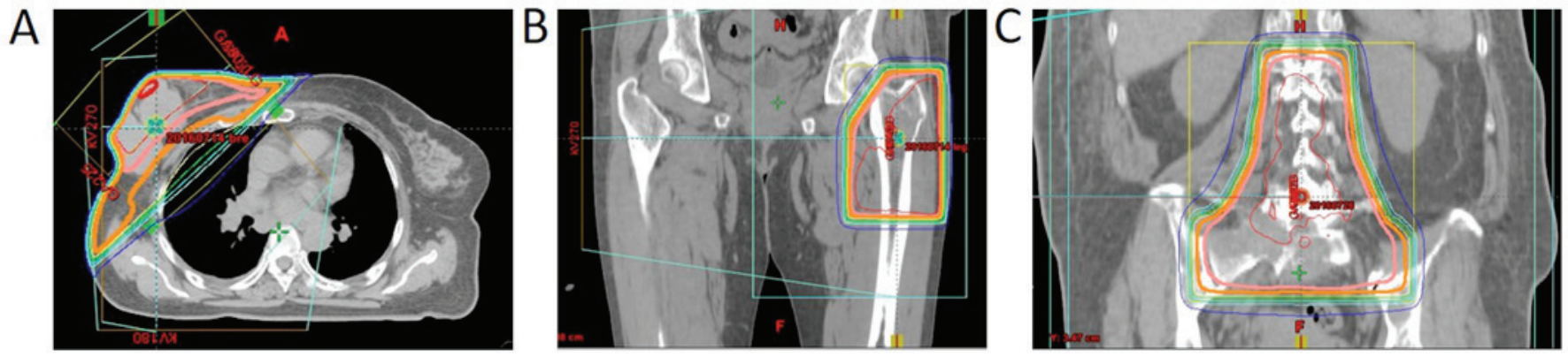

Figure 3. Distribution of the external radiotherapy. The radiation dose was (A) 60 Gy to the right breast, (B) 28 Gy to the left femur and (C) 39 Gy to the lumbar vertebrae and sacrum.

cell surface molecules are translocated and damage-associated molecular pattern molecules are extracellularly released. As a result, ICD occurs and cytotoxic T-lymphocytes (CTLs) are activated. Dendritic cells are activated and induce a systemic antitumor response outside the irradiation field. Suzuki et al reported that chemoradiation induced T-cell recognition of HLA class I antigen-derived peptides in a patient with esophageal squamous cell carcinoma (14). Yoshimoto et al (15) reported that irradiation significantly delayed tumor growth and prolonged median survival time, but depletive mouse tumor models administered anti-CD8 antibody exhibited decreased therapeutic efficacy of irradiation. Radiotherapy is not only employed to destroy the DNA of tumor cells, but also activates tumor-specific immune response and plays a key role in systemic antitumor effect.

The abscopal effect caused by radiation monotherapy is quite rare and has not been extensively investigated. However, with the recent development and use of immunotherapy strategies incorporating combinations of targeted immunomodulators and immune checkpoint blockade with radiotherapy, the abscopal effect has become relevant and clinically meaningful in breast cancer. Dewan et al (16) used a preclinical breast cancer model to show that radiotherapy and CTLA4 blockade induces tumor-specific T-cell responses, not only at the irradiation site, but also outside the radiation field. Clinical trials of immunotherapy using CTLA4 inhibitors or programmed death 1/programmed death ligand inhibitors combined with radiotherapy are ongoing in metastatic breast cancer (17).

In the present study the abscopal effect in a breast cancer patient with multiple bone, lung and lymph node metastases who received palliative radiotherapy is reported. Radiotherapy is an effective strategy, not only for alleviating pain and neurological disorders, but also for eliciting an antitumor response in distant metastases via the abscopal effect. As a result, the quality of life (QOL) of patients with extensive metastases may significantly improve. Systemic medical therapy is more effective compared with local therapy, such as radiotherapy and surgery, for metastatic breast cancer. However, local radiotherapy may be one of the most effective strategies for managing local symptoms for metastatic breast cancer patients to prolong survival while maintaining QOL. Radiotherapy may achieve a systemic antitumor effect in the whole body, very similar to that of chemotherapy, via tumor-specific immune response in breast cancer. Radiotherapy may be a better option for patients with poor general condition, such as the patient in the present case, who may be unable to tolerate chemotherapy. Furthermore, the combination of radiotherapy 
with novel drugs, such as immune checkpoint inhibitors, may represent an effective therapeutic option.

\section{Acknowledgements}

Not applicable.

\section{Funding}

No funding was received.

\section{Availability of data and materials}

Not applicable.

\section{Authors' contributions}

AA managed the therapy for this patient and drafted the manuscript. NA provided palliative therapy for this patient. YA and IS provided radiotherapy for this patient. AS conducted follow-up on the patient in the outpatient clinic. YT, TA and YT gave technical support and conceptual advice. KT contributed to the critical review and supervised the entire work. TO interpreted the data and provided strategy of therapy for this patient and gave final approval for the article. All authors have read and approved the final version of this manuscript.

\section{Ethics approval and consent to participate}

Not applicable.

\section{Patient consent for publication}

Written informed consent was obtained from the patient regarding the publication of the case details and accompanying images.

\section{Competing interests}

The authors declare that they have no competing interests.

\section{References}

1. Wattenberg MM, Fahim A, Ahmed MM and Hodge JW: Unlocking the combination: Potentiation of radiation-induced antitumor responses with immunotherapy. Radiat Res 182: 126-138, 2014

2. Mole RH: Whole body irradiation; radiobiology or medicine? $\mathrm{Br}$ J Radiol 26: 234-241, 1953.
3. Bramhall RJ, Mahady K and Peach AH: Spontaneous regression of metastatic melanoma - clinical evidence of the abscopal effect. Eur J Surg Oncol 40: 34-41, 2014.

4. Okwan-Duodu D, Pollack BP, Lawson D and Khan MK: Role of radiation therapy as immune activator in the era of modern immunotherapy for metastatic malignant melanoma. Am J Clin Oncol 38: 119-125, 2015.

5. Hidaka Y, Takeichi T, Ishikawa Y, Kawamura M and Akiyama M: Abscopal effect of local irradiation treatment for diffuse large B-cell lymphoma. Acta Derm Venereol 97: 1140-1141, 2017.

6. Ishiyama H, Teh BS, Ren H, Chiang S, Tann A, Blanco AI, Paulino AC and Amato R: Spontaneous regression of thoracic metastases while progression of brain metastases after stereotactic radiosurgery and stereotactic body radiotherapy for metastatic renal cell carcinoma: Abscopal effect prevented by the blood-brain barrier? Clin Genitourin Cancer 10: 196-198, 2012.

7. Nakanishi M, Chuma M, Hige S and Asaka M: Abscopal effect on hepatocellular carcinoma. Am J Gastroenterol 103: 1320-1321, 2008.

8. Ohba K, Omagari K, Nakamura T, Ikuno N, Saeki S, Matsuo I, Kinoshita H, Masuda J, Hazama H, Sakamoto I, et al: Abscopal regression of hepatocellular carcinoma after radiotherapy for bone metastasis. Gut 43: 575-577, 1998.

9. Cong Y, Shen G, Wu S and Hao R: Abscopal regression following SABR for non-small-cell-lung cancer: A case report. Cancer Biol Ther 18: 1-3, 2017.

10. Siva S, Callahan J, MacManus MP, Martin O, Hicks RJ and Ball DL: Abscopal [corrected] effects after conventional and stereotactic lung irradiation of non-small-cell lung cancer. J Thorac Oncol 8: e71-e72, 2013

11. Takaya M, Niibe Y, Tsunoda S, Jobo T, Imai M, Kotani S, Unno N and Hayakawa K: Abscopal effect of radiation on toruliform para-aortic lymph node metastases of advanced uterine cervical carcinoma--a case report. Anticancer Res 27 (1B): 499-503, 2007.

12. Takahashi T, Machida K, Honda N, et al: Effective radiotherapy and an abscopal effect for bone metastases of an unknown origin. Jpn J Tomogr 30: 42-45, 2003.

13. Golden EB, Pellicciotta I, Demaria S, Barcellos-Hoff MH and Formenti SC: The convergence of radiation and immunogenic cell death signaling pathways. Front Oncol 2: 88, 2012.

14. Suzuki Y, Mimura K, Yoshimoto Y, Watanabe M, Ohkubo Y, Izawa S, Murata K, Fujii H, Nakano T and Kono K: Immunogenic tumor cell death induced by chemoradiotherapy in patients with esophageal squamous cell carcinoma. Cancer Res 72: 3967-3976, 2012.

15. Yoshimoto Y, Suzuki Y, Mimura K, Ando K, Oike T, Sato H, Okonogi N, Maruyama T, Izawa S, Noda SE, et al: Radiotherapy-induced anti-tumor immunity contributes to the therapeutic efficacy of irradiation and can be augmented by CTLA-4 blockade in a mouse model. PLoS One 9: e92572, 2014

16. Dewan MZ, Galloway AE, Kawashima N, Dewyngaert JK, Babb JS, Formenti SC and Demaria S: Fractionated but not single-dose radiotherapy induces an immune-mediated abscopal effect when combined with anti-CTLA-4 antibody. Clin Cancer Res 15: 5379-5388, 2009.

17. Hu ZI, McArthur HL and Ho AY: The abscopal effect of radiation therapy: What is it and how can we use it in breast cancer? Curr Breast Cancer Rep 9: 45-51, 2017. 